\title{
A quantitative analysis of metal artifact reduction algorithm performance in volume correction with 3 CBCT devices
}

\author{
Karla de Faria Vasconcelos, DDS, MS, PhD, ${ }^{\text {a,b }}$ Polyane Mazucatto Queiroz, DDS, MS, PhD, ${ }^{\mathrm{b}}$ \\ Marina Codari, MSc, PhD, ${ }^{c}$ Laura Ferreira Pinheiro Nicolielo, DDS, MS, ${ }^{a}$ \\ Deborah Queiroz Freitas, DDS, MS, PhD, ${ }^{\mathrm{b}}$ Reinhilde Jacobs, DDS, MS, PhD, ${ }^{\mathrm{a}, \mathrm{d}}$ and \\ Francisco Haiter-Neto, DDS, MS, $\mathrm{PhD}^{\mathrm{b}}$
}

Objective. The aim of this study was to quantitatively assess the performance of metal artifact reduction (MAR) algorithms on the volume of metal cylinders, considering the influence of materials, positions, and fields of view (FOVs), by using 3 cone beam computed tomography (CBCT) devices (NewTom VGi evo, Picasso Trio, and ProMax 3-D Max).

Study Design. Nine phantoms containing cylinders of amalgam, copper-aluminum (CuAl) metal alloy, and titanium, combined in up to 3 positions, were scanned by using 2 different FOVs. MATLAB software was used to evaluate the differences between volumes before and after MAR application, and the possible interference of materials, positions, and FOVs. Wilcoxon's test and the Kruskal-Wallis test were used at a level of significance of $5 \%$.

Results. In general, images containing amalgam and CuAl showed a significant difference in volume before and after MAR application. However, no significant difference after MAR was observed $(P>.05)$ relative to positions and FOVs. MAR had an impact on the cylinder volumes only in the NewTom VGi evo and ProMax 3-D Max scanners.

Conclusions. The performance of MAR algorithms in volume correction of metal objects is dependent on the materials and the CBCT unit. (Oral Surg Oral Med Oral Pathol Oral Radiol 2020;000:1-8)

Cone beam computed tomography (CBCT) may allow for careful 3-dimensional evaluation of the maxillofacial region and improve diagnostic performance. ${ }^{1}$ However, as is the case with other imaging modalities that use ionizing radiation, $\mathrm{CBCT}$ also causes some potential risks to the patient. ${ }^{2}$ Therefore, it is essential that its indication is based on the patient's previous history, the specific purpose of the examination, and knowledge of its limitations, in accordance with the ALADAIP (As Low as Diagnostically Acceptable being Indication-oriented and Patientspecific) principle. ${ }^{3}$

One of the main limitations of CBCT imaging is the generation of artifacts when high-density materials are present in the scanned volume. ${ }^{4}$ Several studies have shown that dental implants, ${ }^{5-9}$ gutta percha, ${ }^{10}$ orthodontic brackets, ${ }^{11}$ and metallic restorations ${ }^{10}$ substantially degrade image quality as a result of the beam hardening phenomenon. ${ }^{9}$ Artifacts hamper the assessment of anatomic structures and

${ }^{a}$ OMFS IMPATH research group, Department of Imaging and Pathology, Faculty of Medicine, University of Leuven and Oral \& Maxillofacial Surgery, University Hospitals Leuven, Leuven, Belgium.

${ }^{\mathrm{b}}$ Division of Oral Radiology, Department of Oral Diagnosis, School of Dentistry of Piracicaba, University of Campinas, Piracicaba, Sao Paulo, Brazil.

${ }^{\mathrm{c}}$ Department of Electronics, Information and Bioengineering, Politecnico di Milano, Milan, Italy.

${ }^{\mathrm{d} D e p a r t m e n t}$ of Dental Medicine, Karolinska Institutet, Huddinge, Sweden.

Received for publication Nov 16, 2019; returned for revision Mar 20, 2020; accepted for publication Mar 31, 2020.

(c) 2020 Elsevier Inc. All rights reserved.

2212-4403/\$-see front matter

https://doi.org/10.1016/j.00oo.2020.03.049 pathologic findings close to the material edges, as occurs with the evaluation of titanium dental implant surfaces, ${ }^{12-14}$ adaptation of crown margins, ${ }^{15}$ and assessment of teeth that have undergone endodontic treatment. ${ }^{16,17}$

One of the available strategies for reducing these artifacts is the use of metal artifact reduction (MAR) algorithms. These algorithms have been extensively tested. ${ }^{18-22}$ However, significant variances have been observed across results obtained with different experimental setups, making the real impact on image interpretability questionable.

Furthermore, many factors, such as object position in the FOV size, ${ }^{23}$ reconstructed field of view, ${ }^{24,25}$ kilovoltage, ${ }^{19}$ and patient motion, may influence image quality. ${ }^{26}$ Additionally, it is a matter of debate as to how these artifacts hinder the assessment of high-density objects, volumes, and their neighboring structures. Moreover, reports on the effective performance of MAR in improving image quality have also provided variable results, and the majority has come from subjective studies.

In this light, the present study aimed to quantitatively assess the performance of MAR on the volume measurements of metal cylinders, considering the influence of the

\section{Statement of Clinical Relevance}

This study confirmed the variability in MAR algorithm performance according to the tested CBCT devices and different types of materials, and the inaccurate performance of all of them compared to a gold standard measurement. 
metal materials, positions of the cylinders, and FOVs by using 3 CBCT devices. The null hypothesis stated that the use of MAR would have no significant effects on the measured volumes of the cylinders as calculated from the CBCT images considering the influence of materials, positions, and FOV.

\section{MATERIAL AND METHODS Phantom manufacturing}

Nine phantoms were created for the project. The phantoms were fabricated by using cylindrical polyvinyl chloride (Tigre, São Paulo, Brazil) molds, measuring $98 \mathrm{~mm}$ in diameter and $40 \mathrm{~mm}$ in height. The molds were filled with acrylic resin (VIP, São Paulo, Brazil) to produce the phantoms and contained spaces for the insertion of 1 to 3 metal cylinders. The cylinders were composed of 3 dental materials commonly used in dental practice: amalgam, copper-aluminum $(\mathrm{CuAl})$ alloy, and titanium. Dental amalgam cylinders were made by using Permite alloy (South Dental Industries, Bayswater, Australia), and $\mathrm{CuAl}$ alloy cylinders were made by using Duracast MS (Dental Gaucho Marquart \& Cia, Barueri, Brazil). Both were fabricated by the researchers according to the manufacturers' instructions. Titanium cylinders were manufactured by the NEODENT Company (Curitiba, Paraná, Brazil). All cylinders had a cross-sectional diameter of $5 \mathrm{~mm}$ and a height of $5 \mathrm{~mm}$.

Before the molds were filled with acrylic resin to complete the creation of phantoms, the positions of the cylinders were carefully checked by means of a comparison microscope (Olympus Optical Co, Ltd., Tokyo, Japan), with the purpose of keeping the cylinders centralized and on the same level. After this, the entire set was kept under 4 bar pressure without heating for 1 hour in a thermal polymerizer to prevent bubble formation in the acrylic resin.

The cylinders were positioned in the phantoms at the vertices of an isosceles triangle. The $\mathrm{ABC}$ positions corresponded to the cylinders placed in the regions of the incisors and second molars (left and right sides). The $\mathrm{AB}$ positions corresponded to the cylinders placed in the regions of the incisors and the left second molars. The B position corresponded to a cylinder placed in the region of the left second molar (Figure 1).

\section{Image acquisitions}

The 9 phantoms were individually positioned in each of the 3 CBCT devices and standardized, according to the guidelines previously demarcated by reference points. Images were acquired by using $\mathrm{CBCT}$ devices that allowed the use of MAR algorithms: NewTom VGi evo (Cefla S.C, Imola, Italy); Picasso Trio (Vatech, Hwaseong, South Korea), and ProMax 3-D Max (Planmeca Oy, Helsinki, Finland). The scanning protocols of the selected devices were comparable.
Two different FOV sizes-medium (M) and small (S) - were selected (Table I). Voxel size was fixed at $0.2 \mathrm{~mm}$ for all CBCT devices, with the aim of excluding possible bias generated by differences in this factor. The 3 variables (materials in the cylinders, positions of the cylinders, and FOVs) were examined by means of 3 different CBCT devices before and after application of MAR algorithms.

Therefore, the combinations of metal materials in the cylinders (3), positions of cylinders in the phantoms (3), FOV sizes (2), number of scanners (3), and use of MAR (2: on and off) yielded a total of 108 images.

\section{Image analyses}

Image segmentation and registration, as well as volume estimation, were performed in MATLAB software (R2016 b, MathWorks, Natick, MA) by a biomedical engineer (M.C.), who used the processing pipeline previously published in the literature. ${ }^{27}$ The segmented volumes of the metal cylinders were calculated as depicted in Figure 2. Considering the known dimensions of the cylinders, the real volume $\left(98.1 \mathrm{~mm}^{3}\right)$ was used as the gold standard.

\section{Statistical analysis}

The measured volume values before and after MAR application were assessed with respect to the effects of materials, positions, and FOVs and also compared with the gold standard. Initially, the data obtained were analyzed for normality by using the Shapiro-Wilk test. As no normal distribution of the data was observed $(P<$ $.05)$, the Kruskal-Wallis test was used to evaluate the effect of applying MAR according to materials and positions, and Wilcoxon's rank-sum test was performed to assess the effect of MAR considering FOV sizes. For comparison with the real cylindrical volumes, 1-way analysis of variance (ANOVA) with Dunnet's post hoc test was used. Analyses were made by using the BioEstat 5.0 software (Fundação Mamiraua, Belém, Brazil). The level of significance for all tests was set at $5 \%$.

\section{RESULTS}

Mean values and standard deviation (M [SD]) of the volumes of the cylinders obtained from the CBCT images considering the different materials (Table II), the position of the cylinders in the phantom (Table III), and the size of the FOV (Table IV) are presented, with the $P$ values derived from the comparison of these volumes with the gold standard.

\section{Effects of metals}

Notwithstanding the different materials, significant variances were observed in the measurement of the volume of the materials for the images obtained in the 


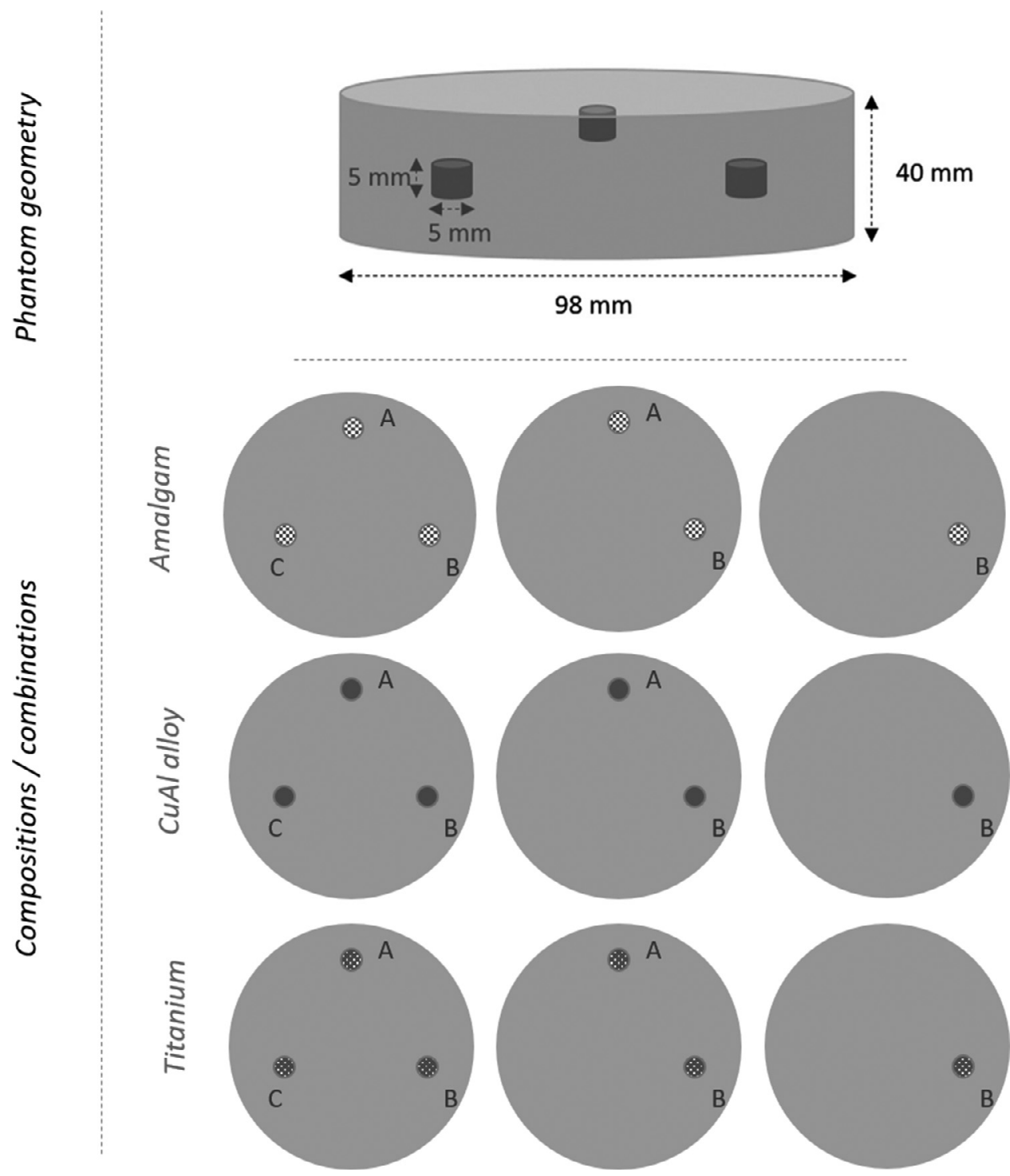

Fig. 1. Diagram illustrating the geometry of the phantom with all possible compositions of the materials and combinations (ABC, $\mathrm{AB}$, and $\mathrm{B}$ ) of positions.

Table I. Acquisition protocols for CBCT images

\begin{tabular}{lllll}
\hline & FOV $(\mathrm{cm})$ Medium/Small & $k V p$ & $m A$ & Exposure time $(s)$ \\
\hline NewTom VGi evo & $10 \times 5 / 5 \times 5$ & 110 & 3.0 & 1.8 \\
Picasso Trio & $8 \times 5 / 5 \times 5$ & 90 & 4.0 & 24 \\
ProMax 3-D Max & $10 \times 5 / 5 \times 5$ & 96 & 5.6 & 12 \\
\hline
\end{tabular}

$C B C T$, cone beam computed tomography; $F O V$, field of view; $k V p$, kilovolt peak; $m A$, milliampere.

NewTom VGi evo $(P=.001)$, Picasso Trio $(P<.001)$, and ProMax 3-D Max $(P=.001)$ devices in the absence of the MAR correction. There were significant differences in the cylinder volume of the images obtained in the NewTom VGi evo $(P=.014)$, Picasso Trio $(P=$ $.003)$, and ProMax 3-D Max $(P=.001)$ devices with the implementation of MAR.
For the NewTom VGi evo device, the measured volumes of amalgam and $\mathrm{CuAl}$ cylinders presented a significant difference compared with the gold standard without MAR $(P<.001)$ and with MAR $(P \leq .031)$, with an increase of up to $19 \%$ (before MAR) and 16\% (after MAR). In contrast, for titanium cylinders, no significant difference was observed $(P=.100)$ in the absence of the 


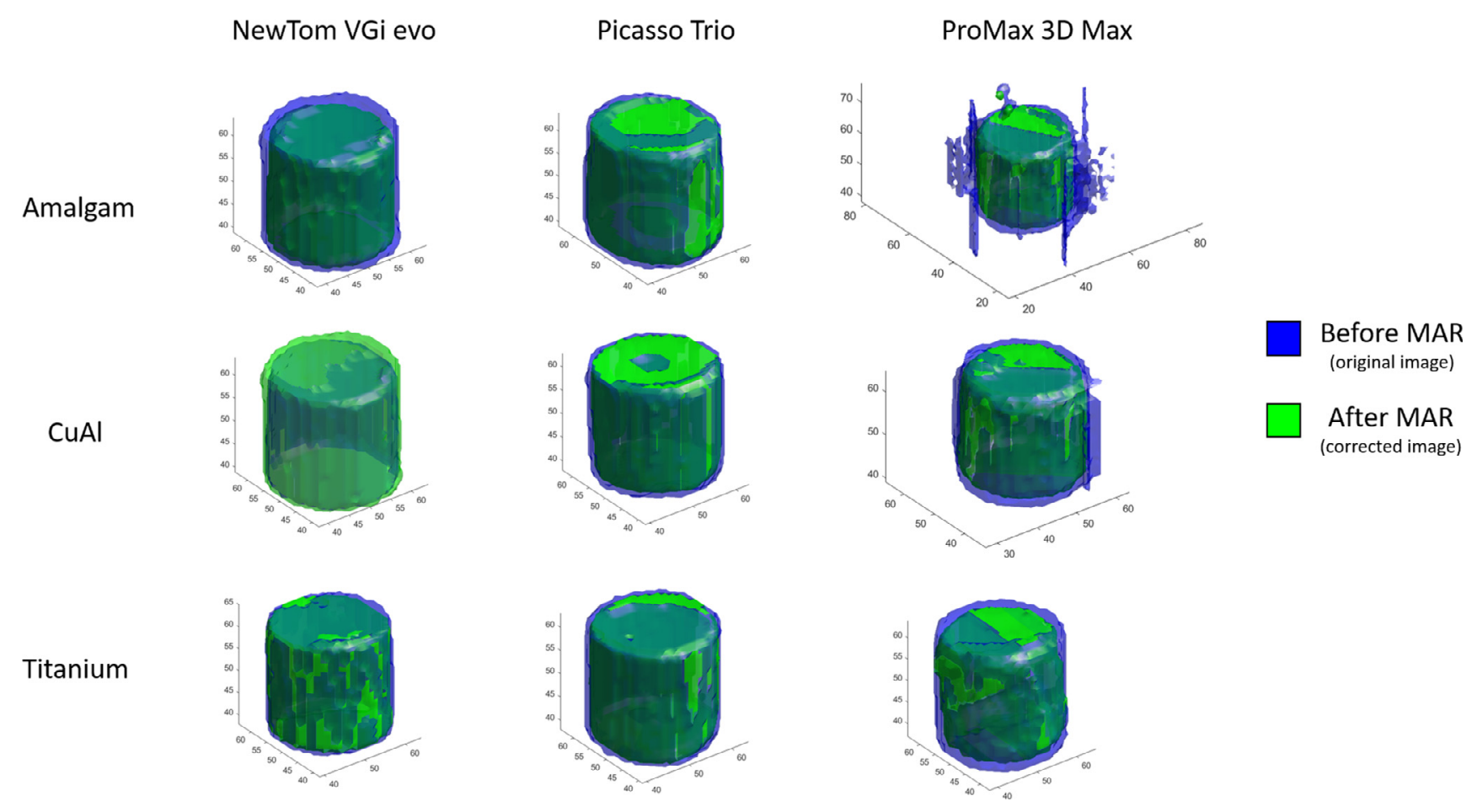

Fig. 2. An illustration of how the volumes of segmented metal cylinders in position B were calculated before (original in blue) and after (corrected in green) MAR application in images of the $\mathrm{ABC}$ group. Axis unit measurement values are expressed in voxels. 
Table II. Mean values and standard deviation (M [SD]) of the measured volumes of the cylinders of different materials, for each CBCT device, with the $P$ values derived from the comparison of these volumes with the gold standard $\left(98.1 \mathrm{~mm}^{3}\right)$

\begin{tabular}{|c|c|c|c|c|c|c|c|c|c|c|c|c|}
\hline & \multicolumn{4}{|c|}{ NewTom VGi evo } & \multicolumn{4}{|c|}{ Picasso Trio } & \multicolumn{4}{|c|}{ ProMax 3-D Max } \\
\hline & \multicolumn{2}{|c|}{ No MAR } & \multicolumn{2}{|c|}{$M A R$} & \multicolumn{2}{|c|}{ No MAR } & \multicolumn{2}{|c|}{$M A R$} & \multicolumn{2}{|c|}{ No MAR } & \multicolumn{2}{|c|}{ MAR } \\
\hline & $M(S D)$ & $P$ value & $M(S D)$ & $P$ value & $M(S D)$ & $P$ value & $M(S D)$ & $P$ value & $M(S D)$ & $P$ value & $M(S D)$ & $P$ value \\
\hline Amalgam & $114.1(2.14)^{*}$ & $<.001$ & $86.6(1.86)^{*}$ & $<.001$ & $116.6(1.21)^{*}$ & $<.001$ & $117.6(1.11)^{*}$ & $<.001$ & $153.1(6.81)^{*}$ & $<.001$ & $134.8(5.25)^{*}$ & $<.001$ \\
\hline $\mathrm{CuAl}$ & $94.1(1.33)^{*}$ & $<.001$ & $106.3(10.11)^{*}$ & .031 & $98.9(1.65)$ & .174 & $98.8(1.72)$ & .263 & $124.1(1.72)^{*}$ & $<.001$ & $116.1(2.23)^{*}$ & $<.001$ \\
\hline Titanium & $99.1(0.98)$ & .100 & $86.1(0.75)^{*}$ & .012 & $104.6(1.3)^{*}$ & $<.001$ & $98.6(1.51)$ & .328 & $119.6(3.5)^{*}$ & $<.001$ & $113.6(3.27)^{*}$ & $<.001$ \\
\hline
\end{tabular}

*Significant difference in comparison with gold standard, considering level of significance of $5 \%$.

All values are expressed as $\mathrm{mm}^{3}$.

CBCT, cone beam computed tomography.

Table III. Mean values and standard deviation (M [SD]) of the measured volumes of the cylinders calculated from images obtained with the cylinders in different positions, for each CBCT device, with the $P$ values derived from the comparison of these volumes with the gold standard $\left(98.1 \mathrm{~mm}^{3}\right)$

\begin{tabular}{|c|c|c|c|c|c|c|c|c|c|c|c|c|}
\hline & \multicolumn{4}{|c|}{ NewTom VGi evo } & \multicolumn{4}{|c|}{ Picasso Trio } & \multicolumn{4}{|c|}{ ProMax 3-D Max } \\
\hline & \multicolumn{2}{|c|}{ No MAR } & \multicolumn{2}{|c|}{$M A R$} & \multicolumn{2}{|c|}{ No MAR } & \multicolumn{2}{|c|}{$M A R$} & \multicolumn{2}{|c|}{ No MAR } & \multicolumn{2}{|c|}{ MAR } \\
\hline & $M(S D)$ & $P$ value & $M(S D)$ & P value & $M(S D)$ & Pvalue & $M(S D)$ & $P$ value & $M(S D)$ & $P$ value & $M(S D)$ & $P$ value \\
\hline$\overline{\mathrm{ABC}}$ & $101.61(9.05)$ & .353 & $95.00(13.67)$ & .601 & $106.08(8.54)^{*}$ & .039 & $104.08(9.51)$ & .179 & $132.99(14.89)^{*}$ & .002 & $123.01(11.39)^{*}$ & .003 \\
\hline $\mathrm{AB}$ & $103.31(9.46)$ & .236 & $94.61(12.03)$ & .509 & $106.98(8.07)^{*}$ & .035 & $105.16(9.15)$ & .107 & $134.33(19.98)^{*}$ & .007 & $120.11(11.25)^{*}$ & .005 \\
\hline B & $103.32(9.77)$ & .225 & $90.76(8.01)$ & .053 & $107.72(8.39)^{*}$ & .016 & $105.33(8.99)$ & .097 & $129.41(14.27)^{*}$ & .003 & $121.57(9.93)^{*}$ & .002 \\
\hline
\end{tabular}

*Significant difference in comparison with gold standard, considering level of significance of $5 \%$.

All values are expressed as $\mathrm{mm}^{3}$.

$C B C T$, cone beam computed tomography. 
MAR tool, whereas the presence of the MAR resulted in a significant reduction $(P=.012)$ of the cylinder volume, in relation to the real volume (see Table II).

For the Picasso Trio device, compared with the gold standard, the volumes of amalgam cylinders were overestimated $(P<.001)$ by up to $20 \%$, whether or not MAR algorithms were applied $(P<.001)$. For the CuAl cylinders, no significant differences in volume was observed in the presence $(P=.174)$ or absence $(P=.263)$ of MAR. For titanium cylinders, in the images before MAR, an overestimation of up to $7 \%$ in the volume of the cylinder $(P<.001)$ was observed; however, after MAR, no significant difference was found $(P=.328)$, as shown in Table II.

For the ProMax 3-D Max device, compared with the gold standard, significant overestimation was observed for all the materials, ranging from $13 \%$ (titanium) to $67 \%$ (amalgam), irrespective of application of MAR algorithms $(P<.001)$, as listed in Table II.

\section{Effects of positions}

In the absence of MAR, there were no significant differences in the images obtained with the cylinders in the different positions for the NewTom VGi evo $(P=.804)$, Picasso Trio $(P=.834)$, and ProMax 3-D Max $(P=.824)$ devices. With application of MAR algorithm, there were no significant differences in relation to the volumes calculated from the images obtained with the cylinders in different positions for the NewTom VGi evo $(P=.875)$, Picasso Trio $(P=.587)$, and ProMax 3-D Max $(P=.519)$.

However, in relation to the gold standard (see Table III), there were significant differences in the volumes obtained from the Picasso Trio images without MAR $(P \leq .039)$ and the ProMax 3-D Max images without and with MAR $(P \leq .007)$ for all cylinder positions. There was an increase in the cylinder volume obtained from all these images.

\section{Effects of FOV}

Without application of MAR algorithms, there were no differences observed in the volume obtained from the images with different FOV sizes in the NewTom VGi evo $(P=.535)$, Picasso Trio $(P=.353)$, and ProMax 3-D Max $(P=.627)$ devices. With application of MAR algorithms, there were no significant differences in the volume calculated from the images obtained with different FOV sizes for the NewTom VGi evo $(P=.563)$, Picasso Trio $(P=.123)$, and ProMax 3-D Max $(P=.753)$ devices.

However, in relation to the gold standard, there were significant differences in the volumes obtained both without and with MAR from the images of Picasso Trio $(P \leq .023)$ and ProMax 3-D Max $(P<.001)$ for both sizes of FOV (see Table IV). There was an increase in the cylinder volume obtained from all these images. 


\section{DISCUSSION}

In general, methods at present available for metal artifact correction may be divided into 2 types: (1) interactive methods and (2) projection completion methods. ${ }^{28,29}$ These methods have shown advantages and disadvantages in terms of computational simplicity, precision, and efficiency in the reduction of metal artifacts. Different characteristics of these methods may result in different algorithm performances, making it difficult, and sometimes impossible, to compare them. In the present study, we did not obtain detailed information about the MAR algorithms used by the manufacturers. However, we observed that those we tested performed in different ways, depending on the CBCT device.

A previous study showed evidence that the extent of volumetric distortion artifact varied across different types of CBCT devices. ${ }^{16}$ This effect, also known as "blooming," must be taken into careful consideration during endodontic and implant dentistry diagnosis in terms of evaluating the volumes of dense structures and in terms of compromising the evaluation of adjacent structures. ${ }^{30}$ Our study allowed for the volumetric quantification of the blooming artifact caused by metallic materials and the evaluation of the performance of some MAR algorithms available on the market.

In terms of segmented volume, application of MAR algorithms had some impact on the NewTom VGi evo and ProMax 3-D Max images. However, none of the algorithms was capable of completely removing distortion of the object compared with the real object volume. In the NewTom VGi evo device, even after correction, the amalgam and titanium cylinders were overestimated by up to $14 \%$, and the $\mathrm{CuAl}$ cylinders were overestimated by up to $16 \%$ of the real volume. In the ProMax 3-D Max device, after MAR application, an increase in volume values of up to $42 \%$ was discovered in the amalgam and $\mathrm{CuAl}$ alloy cylinders. Additionally, we sometimes observed an overcorrection, resulting in a huge difference between segmented and real volume values.

With regard to the Picasso Trio images, application of MAR algorithms seemed to have no influence on the estimation of cylindrical volumes. Indeed, MAR algorithms could hardly add value because there seemed to be less distortion. Even in the uncorrected images, the results were comparable with the gold standard measures for $\mathrm{CuAl}$ alloy and titanium, corroborating the findings of previous studies. ${ }^{31,32}$

With regard to the different materials, images of amalgam (for all the devices) and $\mathrm{CuAl}$ (for NewTom VGi evo and ProMax 3-D Max) showed a significant overestimation of volume values both before and after application of MAR algorithms. All MAR algorithms exhibited their best performance when the image was highly corrupted by artifacts, as it was in the amalgam and $\mathrm{CuAl}$ images. This evidence has also been verified in previous studies. ${ }^{21}$ Similar behavior was observed in relation to the background of the image in a previous study. ${ }^{22}$ The literature also shows that different materials result in different patterns and intensities of artifacts. ${ }^{32}$ The higher the atomic number of the material, the greater will be the expression of the artifact, and consequently, the better will be the performance of MAR in the correction of artifacts.

Our results demonstrated that cylinder positions and FOVs did not impact the performance of the MAR algorithms tested. A different result was found in 1 study $;^{33}$ however, those authors used a different approach by evaluating only 1 high-density material and by using SD values measured in axial images.

The MAR algorithms currently available on the market for CBCT image processing are constantly undergoing improvement. Through this study, it was possible to confirm the existing variability of MAR performance in volume correction. The positions of the objects and the FOV size had no impact on the performance of MAR algorithms in terms of volume correction.

\section{CONCLUSIONS}

The performance of the tested MAR algorithms in correcting metal artifact depended on the CBCT device and the material used. Among the devices studied, application of MAR algorithms is recommended for NewTom VGi evo and ProMax 3-D Max scans containing materials with high atomic numbers.

\section{ACKNOWLEDGMENT}

We would like to thank NEODENT Company (Curitiba, Paraná, Brazil) for providing the titanium cylinders for the present research (Protocol \# 0309/14). During her $\mathrm{PhD}$ studies, M.C. contributed to the development of the Cefla S.C. metal artifact reduction algorithm.

\section{REFERENCES}

1. Jacobs R, Salmon B, Codari M, Hassan B, Bornstein MM. Cone beam computed tomography in implant dentistry: recommendations for clinical use. BMC Oral Health. 2018;18:88.

2. Pauwels R, Beinsberger J, Collaert B, et al. SEDENTEXCT Project Consortium. Effective dose range for dental cone beam computed tomography scanners. Eur J Radiol. 2012;81:267-271.

3. Oenning AC, Pauwels R, Stratis A, et al. Dimitra research group. Halve the dose while maintaining image quality in paediatric cone beam CT. Sci Rep. 2019;9:5521.

4. Schulze R, Heil U, Gross D, et al. Artifacts in TCFC: a review. Dentomaxillofac Radiol. 2011;40:265-273.

5. Draenert FG, Coppenrath E, Herzog P, Müller S, Mueller-Lisse UG. Beam hardening artifacts occur in dental implant scans with the NewTom cone beam CT but not with the dental 4-row multidetector CT. Dentomaxillofac Radiol. 2007;36:198-203.

6. Schulze RK, Berndt D, d'Hoedt B. On cone-beam computed tomography artifacts induced by titanium implants. Clin Oral Implants Res. 2010;21:100-107. 
7. Chindasombatjaroen J, Kakimoto N, Murakami S, Maeda Y, Furukawa S. Quantitative analysis of metallic artifacts caused by dental metals: comparison of cone-beam and multi-detector row CT scanners. Oral Radiol. 2011;27:114-120.

8. Esmaeili F, Johari M, Haddadi P, Vatankhah M. Beam hardening artifacts: comparison between two cone beam computed tomography scanners. J Dent Res Dent Clin Dent Prospects. 2012;6:49-53.

9. Pauwels R, Stamatakis H, Bosmans H, et al. Quantification of metal artifacts on cone beam computed tomography images. Clin Oral Implants Res. 2013;100:94-99.

10. Klinke T, Daboul A, Maron J, et al. Artifacts in magnetic resonance imaging and computed tomography caused by dental materials. PLoS One. 2012;7:E31766.

11. Sanders MA, Hoyjberg C, Chu CB, Leggitt VL, Kim JS. Common orthodontic appliances cause artifacts that degrade the diagnostic quality of TCFC images. $J$ Calif Dent Assoc. 2007;35:850-857.

12. Kamburŏglu K, Kolsuz E, Murat S, Eren H, Yüksel S, Paksoy CS. Assessment of buccal marginal alveolar peri-implant and periodontal defects using a cone beam CT system with and without the application of metal artifact reduction mode. Dentomaxillofac Radiol. 2013;42:20130176.

13. de-Azevedo-Vaz SL, Alencar PN, Rovaris K, Campos PS, Haiter-Neto F. Enhancement cone beam computed tomography filters improve in vitro periimplant dehiscence detection. Oral Surg Oral Med Oral Pathol Oral Radiol. 2013;116:633-639.

14. de-Azevedo-Vaz SL, Vasconcelos Kde F, Neves FS, Melo SL, Campos PS, Haiter-Neto F. Detection of periimplant fenestration and dehiscence with the use of two scan modes and the smallest voxel sizes of a cone-beam computed tomography device. Oral Surg Oral Med Oral Pathol Oral Radiol. 2013;115:121-127.

15. Carneiro VC, Siqueira CA, Valentim FB, et al. Accuracy of three cone beam computed tomography systems in the detection of implant-abutment misfit. Int J Prosthodont. 2019;32:198-200.

16. Celikten B, Jacobs R, de Faria Vasconcelos K, et al. Comparative evaluation of cone beam CT and micro-CT on blooming artifacts in human teeth filled with bioceramic sealers. Clin Oral Investig. 2019;23:3267-3273.

17. Neves FS, Freitas DQ, Campos PS, Ekestubbe A, Lofthag-Hansen S. Evaluation of cone-beam computed tomography in the diagnosis of vertical root fractures: the influence of imaging modes and root canal materials. J Endod. 2014;40:1530-1536.

18. Mahnken AH, Raupach R, Wildberger JE, et al. New algorithm for metal artifact reduction in computed tomography: in vitro and in vivo evaluation after total hip replacement. Invest Radiol. 2003;38:769-775.

19. Bechara BB, Moore WS, McMahan CA, Noujeim M. Metal artifact reduction with cone beam CT: an in vitro study. Dentomaxillofac Radiol. 2012;41:248-253.

20. Bezerra IS, Neves FS, Vasconcelos TV, Ambrosano GM, Freitas DQ. Influence of the artifact reduction algorithm of Picasso Trio CBCT system on the diagnosis of vertical root fractures in teeth with metal posts. Dentomaxillofac Radiol. 2015;44:20140428.

21. Queiroz PM, Oliveira ML, Groppo FC, Haiter-Neto F, Freitas DQ. Evaluation of metal artifact reduction in cone-beam computed tomography images of different dental materials. Clin Oral Investig. 2018;22:419-423.

22. Vasconcelos KF, Codari M, Queiroz PM, et al. The performance of metal artifact reduction algorithms in cone beam computed tomography images considering the effects of materials, metal positions, and fields of view. Oral Surg Oral Med Oral Pathol Oral Radiol. 2019;127:71-76.

23. Pauwels R, Jacobs R, Bogaerts R, Bosmans H, Panmekiate S. Reduction of scatter-induced image noise in cone beam computed tomography: effect of field of view size and position. Oral Surg Oral Med Oral Pathol Oral Radiol. 2016;121:188-195.

24. Oliveira ML, Tosoni GM, Lindsey DH, Mendoza K, Tetradis S, Mallya SM. Influence of anatomical location on CT numbers in cone beam computed tomography. Oral Surg Oral Med Oral Pathol Oral Radiol. 2013;115:558-564.

25. Brüllmann D, Schulze RK. Spatial resolution in CBCT machines for dental/maxillofacial applications-what do we know today? Dentomaxillofac Radiol. 2015;44:20140204.

26. Spin-Neto R, Mudrak J, Matzen LH, Christensen J, Gotfredsen E, Wenzel A. Cone beam CT image artifacts related to head motion simulated by a robot skull: visual characteristics and impact on image quality. Dentomaxillofac Radiol. 2013;42:32310645.

27. Codari M, de Faria Vasconcelos K, Ferreira Pinheiro Nicolielo L, Haiter Neto F, Jacobs R. Quantitative evaluation of metal artifacts using different $\mathrm{CBCT}$ devices, high-density materials and field of views. Clin Oral Implants Res. 2017;28:1509-1514.

28. De Man B, Nuyts J, Dupont P, Marchal G, Suetens P. An iterative maximum-likelihood polychromatic algorithm for CT. IEEE Trans Med Imaging. 2001;20:999-1008.

29. Van Slambrouck K, Nuyts J. Metal artifact reduction in computed tomography using local models in an image block-iterative scheme. Med Phys. 2012;39:7080-7093.

30. Vanderstuyft T, Tarce M, Sanaan B, Jacobs R, De Faria Vasconcelos K, Quirynen M. Inaccuracy of buccal bone thickness estimation on cone-beam CT due to implant blooming: an ex-vivo study. J Clin Periodontol. 2019;46:1134-1143.

31. Freitas DQ, Fontenele RC, Nascimento EHL, Vasconcelos TV, Noujeim M. Influence of acquisition parameters on the magnitude of cone beam computed tomography artifacts. Dentomaxillofac Radiol. 2018;47:20180151.

32. Helvacioglu-Yigit D, Demirturk Kocasarac H, Bechara B, Noujeim M. Evaluation and reduction of artifacts generated by 4 different root-end filling materials by using multiple cone-beam computed tomography imaging settings. J Endod. 2016;42:307314.

33. Queiroz PM, Santaella GM, da Paz TD, Freitas DQ. Evaluation of a metal artifact reduction tool on different positions of a metal object in the FOV. Dentomaxillofac Radiol. 2017;46:20160366.

\section{Reprint requests:}

Karla de Faria Vasconcelos
OMFS IMPATH Research Group
Department of Imaging \& Pathology
Faculty of Medicine
University of Leuven
Campus Sint-Rafael
Kapucijnenvoer 33
3000 Leuven
Belgium.
Karlafav13@gmail.com

